\section{Single-molecule DNA sequencing of acute myeloid leukemia and myelodysplastic syndromes with multiple TP53 alterations}

Although the frequency of TP53 mutations in hematologic malignancies is low, these mutations have a high clinical relevance and are usually associated with poor prognosis. Somatic TP53 mutations have been detected in up to $73.3 \%$ of cases of acute myeloid leukemia (AML) with complex karyotype and $18.9 \%$ of AML with other unfavorable cytogenetic risk factors. ${ }^{1}$ AML with TP53 mutations, and/or chromosomal aneuploidy, has been defined as a distinct AML subtype. In low-risk myelodysplastic syndromes (MDS), TP53 mutations occur at an early disease stage and predict disease progression. $^{2}$ TP53 mutation diagnosis is now part of the revised European LeukemiaNet (ELN) guidelines. ${ }^{3,4}$

The use of next generation sequencing (NGS), particularly ultra-deep sequencing, has led to the discovery that patients with either MDS or AML (either de novo, secondary or therapy-related) present multiple TP53 mutations, suggesting that several TP53 independent clones may coexist. ${ }^{5}$ Patient follow up also reveals a highly dynamic evolution of these mutations during disease progression in treated and untreated patients. ${ }^{6,7}$ This observation is in line with the recent recognition that human tumors harbor an extensive genetic intratumoral heterogeneity. ${ }^{8}$ These findings will likely have implications for therapy and biomarker discovery, and determination of genetic complexity is becoming part of clinical decision-making processes in the age of precision medicine.

In this report, in silico analysis of the UMD_TP53 database showed that TP53 variants detected in patients with multiple TP53 alterations are fully oncogenic. Furthermore, using long-range single-molecule real-time (SMRT) sequencing on AML and MDS patients harboring multiple TP53 mutations, we showed that all of these variants are localized on different subclones, emphasizing the considerable tumor heterogeneity in these patients.

The 2017 release of the UMD_TP53 database contains the mutation status of 75,448 patients, including 922 cases of AML and 899 cases of MDS (Online Supplementary Table S1). ${ }^{9,10}$ Among these patients, 158 MDS cases $(22.3 \%)$ and 99 AML cases $(13 \%)$ harbor more than one TP53 variant in their tumors, higher than the rate observed in solid tumors (Online Supplementary Table S1). Chronic Lymphocytic Leukemia (CLL) patients also harbor a high frequency of tumors with multiple TP53 mutations. This feature has been observed with increasing frequency over recent years with the advent of deep sequencing techniques. Whether or not all of the multiple variants identified in these patients are truly deleterious or comprise a mix of driving and passenger mutations has never been addressed. The UMD_TP53 database includes quantitative functional data for all TP53 missense variants and can therefore be used to determine whether patients with multiple TP53 mutations frequently harbor non-deleterious TP53 variants. Analysis of the $257 \mathrm{AML}$ and MDS cases with more than one TP53 variant showed that the majority (98\%) of these variants are true deleterious TP53 mutations with complete loss of function and not simply random passenger mutations co-selected during tumor progression (Online Supplementary Table S2 and Online Supplementary Figure $S 1$ a to e). A few non-deleterious variants have been identified, but they are likely very rare non-somatic polymorphisms.
To further demonstrate the presence and the dynamics of multiple independent tumor clones in AML and MDS, we have developed a novel, third-generation single-molecule real-time (SMRT) sequencing assay using the Pacific Biosciences platform with long-read lengths that span the most frequently mutated region of the TP53 gene. Sanger sequencing cannot be used to define the allelic distribution of multiple TP53 variants. This is also true for standard NGS if the two variants are more than 200 base pairs apart. On the other hand, SMRT analysis can be used to phase mutations located multiple kilobases apart directly from sequencing reads.

Eleven patients harboring multiple TP53 mutations in their tumors were enrolled. For 3 patients, sequential samples were available to assess the evolution of the various variants. The TP53 status of these patients was already defined according to stringent clinical criteria using either Sanger sequencing or standard NGS (Online Supplementary Material and Online Supplementary Table S3). In silico analysis of all these variants using the UMD_TP53 database showed that they were true deleterious TP53 mutations that have already been described in various types of cancer (Online Supplementary Table S1).

The majority of mutations detected for clinical evaluation were readily identified by SMRT, except for 2 variants that were not included in the amplicon used for analysis. SMRT identified 5 mutations that were not identified by clinical analysis (Online Supplementary Table S3 and Online Supplementary Figure S2). Manual examination of the sequencing data performed for clinical analysis confirmed that 2 of these mutations were detected at a frequency below the cut-off used for the analysis (Table 1). Most of the remaining mutations detected by SMRT were present at a very low frequency (Online Supplementary Figure S2). The variant allele frequency (VAF) observed for each variant detected by the two analyses was remarkably similar, despite being performed in different centers according to very different methodologies (Online Supplementary Figure S3).

Our analysis shows that all oncogenic TP53 variants were located in different alleles (Table 1 and Online Supplementary Figures $S 4 a$ to $S 4 k$ ). For two samples, patient Fr7, sample $7 \mathrm{~b}$ and patient Fr2, the close proximity of two TP53 variants allowed analysis of the alignment obtained after standard NGS and confirmed that these mutations were carried by different alleles (Online Supplementary Figures S5 and S6). For 2 samples, the allelic distribution was also confirmed by the observation that the different TP53 variants were associated with different TP53 haplotypes (Online Supplementary Figure $S 4 j$ and $k$, patients Fr10 and Fr11).

Figure 1 shows a typical result observed for two samples collected 5 years apart from a patient with multiple TP53 mutations. Of note, the diagnostic sample was negative with standard NGS and was therefore not used for SMRT analysis. In the first sample analysed by SMRT, clinical analysis identified two pathogenic TP53 mutations confirmed by SMRT. SMRT analysis also identified two novel TP53 mutations at very low frequency and showed that the 4 variants were distributed in different TP53 molecules. The two novel variants were readily identified in the second sample collected 5 years later. New TP 53 variants were also identified by both methodologies ( 2 variants) and 2 additional variants were found at low frequency by SMRT (Figure 1). All these variants, carried by different TP53 molecules, were true driver mutations already identified in multiple tumor types, as shown by their high frequency in the UMD_TP53 data- 
base. This dynamic evolution of the various subclones can also lead to the elimination of certain subclones, as shown for patient Fr10 with the disappearance of TP53 variants (Online Supplementary Figure S4J).
Using both in silico analysis and SMRT sequencing, we demonstrate that the presence of multiple subclones with different TP53 variants is a common feature in AML and MDS. All TP53 variants detected in MDS and AML

Table 1. Patient characteristics. More information is available in Online Supplementary Table S1.

\begin{tabular}{|c|c|c|c|c|c|c|c|c|}
\hline Patient & Sample & $\begin{array}{l}\text { Sex/Age } \\
\text { (diagnosis) }\end{array}$ & $\begin{array}{l}\text { Hematologic } \\
\text { malignancy }^{2}\end{array}$ & Treatment & $\begin{array}{c}\text { TP53 } \\
\text { alteration } \\
\text { (clinical) }\end{array}$ & $\begin{array}{c}\text { TP53 } \\
\text { alteration } \\
\text { (SMRT) }^{3}\end{array}$ & $\begin{array}{c}\text { TP53 } \\
\text { Mutation }\end{array}$ & $\begin{array}{c}\text { Allelic } \\
\text { distribution }\end{array}$ \\
\hline \multirow[t]{2}{*}{ Frl } & Frl & $\mathrm{M} / 77$ & De novo MK-AML & $\begin{array}{l}\text { No (at diagnosis, } \\
\text { before treatment) }\end{array}$ & Yes & Yes & c. $673-2 \mathrm{~A}>\mathrm{G}$ & NR \\
\hline & & & & & Yes & $\mathrm{No}^{6}$ & c.897_912del15 & NR \\
\hline \multirow[t]{2}{*}{ Fr2 } & Fr2 & $\mathrm{M} / 63$ & De novo MK-AML & $\begin{array}{l}\text { No (at diagnosis, } \\
\text { before treatment) }\end{array}$ & Yes & Yes & c. $673-2 \mathrm{~A}>\mathrm{T}$ & Yes \\
\hline & & & & & Yes & Yes & c. $743 \mathrm{G}>\mathrm{A}$ & Yes \\
\hline \multirow[t]{2}{*}{ Fr3 } & Fr3 & $\mathrm{F} / 73$ & De novo MK-AML & $\begin{array}{l}\text { No (at diagnosis, } \\
\text { before treatment) }\end{array}$ & Yes & Yes & c. $413 \mathrm{C}>\mathrm{T}$ & Yes \\
\hline & & & & & Yes & Yes & c.794T $>C$ & Yes \\
\hline \multirow[t]{2}{*}{ Fr4 } & Fr4 & $\mathrm{F} / 78$ & De novo MK-AML & $\begin{array}{l}\text { No (at diagnosis, } \\
\text { before treatment) }\end{array}$ & Yes & Yes & c. $395 \mathrm{~A}>\mathrm{G}$ & Yes \\
\hline & & & & & Yes & Yes & c. $824 \mathrm{G}>\mathrm{T}$ & Yes \\
\hline \multirow[t]{3}{*}{ Fr5 } & Fr5 & $\mathrm{F} / 73$ & De novo MK-AML & No (at diagnosis, & Yes & Yes & c. $637 \mathrm{C}>\mathrm{T}$ & Yes \\
\hline & & & & before treatment) & Yes & Yes & c. $455 \mathrm{C}>\mathrm{T}$ & Yes \\
\hline & & & & & Yes & Yes & c. $455 \mathrm{C}>\mathrm{T}$ & Yes \\
\hline \multirow[t]{6}{*}{ Fr6 } & Fr6 & $\mathrm{F} / 75$ & s-AML (post LR-MDS del5q) & $\begin{array}{l}\text { Yes } \\
\text { (Lenalidomide) }\end{array}$ & Yes & No & c. $524 \mathrm{G}>\mathrm{A}$ & NR \\
\hline & & & & & Yes & Yes & c. $844 \mathrm{C}>\mathrm{T}$ & NR \\
\hline & Fr7a & & & & Yes & Yes & c. $314 \mathrm{G}>\mathrm{T}$ & Yes \\
\hline & & & & & Yes & Yes & c. $743 \mathrm{G}>\mathrm{A}$ & Yes \\
\hline & & & & & No & Yes & c. $818 \mathrm{G}>\mathrm{A}$ & Yes \\
\hline & & & & & No & Yes & c. $742 \mathrm{C}>\mathrm{T}$ & Yes \\
\hline \multirow[t]{8}{*}{$\mathrm{Fr}^{7}$} & & $\mathrm{~F} / 73$ & LR-MDS del5q & Yes & Yes & Yes & c. $314 \mathrm{G}>\mathrm{T}$ & Yes \\
\hline & & & & (Lenalidomide) & Yes & Yes & c. $743 \mathrm{G}>\mathrm{A}$ & Yes \\
\hline & & & & & Yes & Yes & c. $584 \mathrm{~T}>\mathrm{A}$ & Yes \\
\hline & Fr7b & & & & Yes & Yes & c. $614 \mathrm{~A}>\mathrm{G}$ & Yes \\
\hline & & & & & Yes & Yes & c. $818 \mathrm{G}>\mathrm{A}$ & Yes \\
\hline & & & & & Yes & Yes & c. $833 \mathrm{C}>\mathrm{G}$ & Yes \\
\hline & & & & & No & Yes & c. $524 \mathrm{G}>\mathrm{A}$ & Yes \\
\hline & & & & & No & Yes & c. $659 \mathrm{~A}>\mathrm{G}$ & Yes \\
\hline \multirow[t]{3}{*}{ Fr8 } & Fr8 & $\mathrm{F} / 72$ & s-AML (post LR-MDS del5q) & Yes & Yes & Yes & c. $725 \mathrm{G}>\mathrm{T}$ & NR \\
\hline & & & & (Lenalidomide) & Yes & No & c. $920-1 \mathrm{G}>\mathrm{A}$ & NR \\
\hline & $\mathrm{Fr} 9 \mathrm{a}^{8}$ & & s-AML (post LR-MDS del5q) & & Yes & Yes & c. $421 \mathrm{~T}>\mathrm{G}$ & Yes \\
\hline \multirow[t]{3}{*}{ Fr9 } & & $\mathrm{M} / 76$ & & (Lenalidomide) & Yes & Yes & c.711G $>\mathrm{T}$ & Yes \\
\hline & Fr9b & & s-AML (post LR-MDS del5q) & & Yes & Yes & c. $421 \mathrm{~T}>\mathrm{G}$ & Yes \\
\hline & & & & & Yes $^{9}$ & Yes & c. $711 \mathrm{G}>\mathrm{T}$ & Yes \\
\hline \multirow{4}{*}{ Fr10 10} & Fr10a & & LR-MDS del5q & & Yes & Yes & c. $743 \mathrm{G}>\mathrm{A}$ & Yes \\
\hline & & $\mathrm{M} / 69$ & & (Lenalidomide) & Yes & Yes & c. $844 \mathrm{C}>\mathrm{T}$ & Yes \\
\hline & & & & & Yes & Yes & c. $817 \mathrm{C}>\mathrm{T}$ & Yes \\
\hline & Fr10b & & s-AML (post LR-MDS del5q) & $\begin{array}{l}\text { Yes } \\
\text { (Lenalidomide) }\end{array}$ & Yes & Yes & c. $743 \mathrm{G}>\mathrm{A}$ & Yes \\
\hline \multirow{7}{*}{ Fr11 ${ }^{11}$} & & & & & Yes & Yes & c. $844 \mathrm{C}>\mathrm{T}$ & Yes \\
\hline & Frlla & $\mathrm{F} / 85$ & LR-MDS del5q & Yes & Yes $^{7}$ & Yes & c. $659 \mathrm{~A}>\mathrm{G}$ & Yes \\
\hline & & & & (Lenalidomide) & Yes $^{12}$ & Yes & c. $840 \mathrm{~A}>\mathrm{T}$ & NR \\
\hline & & & & & No & Yes & c. $701 \mathrm{~A}>\mathrm{G}$ & Yes \\
\hline & Frllb & $\mathrm{F} / 85$ & LR-MDS del5q & Yes & Yes & Yes & c. $701 \mathrm{~A}>\mathrm{G}$ & Yes \\
\hline & & & & (Lenalidomide) & Yes & Yes & c. $659 \mathrm{~A}>\mathrm{G}$ & Yes \\
\hline & & & & & Yes & Yes & c. $840 \mathrm{~A}>\mathrm{T}$ & Yes \\
\hline
\end{tabular}

'MK-AML: Monosomal karyotype AML; LR-MDS: Lower-risk-MDS; ${ }^{2}$ Identification of TP53 mutations for clinical analysis using conventional Sanger sequencing (VAF cut-off: 10-15\%)or standard NGS (VAF cut-off: 1\%); ${ }^{3}$ Identification of TP53 mutations using SMRT sequencing. ${ }^{4}$ TP53 variant description using the NM_000546.5 reference. A full description of the mutations and their consequences is presented in Online Supplementary Table S1. ${ }^{5}$ Yes: mutations are located on different alleles; NR: not relevant. ${ }^{6}$ Mutation outside the amplicon used for SMRT analysis. ${ }^{7}$ Samples Fr7a and Fr7b were taken at an interval of 59 months. ${ }^{8}$ Sample $9 \mathrm{a}$ and $9 \mathrm{~b}$ : DNA extracted from a frozen pellet from whole blood leukocytes or a cytogenetic pellet from bone marrow, respectively (same sampling date). ${ }^{9}$ Mutation detected by SMRT and identified at very low frequency by reviewing the standard NGS data. ${ }^{10}$ Sample Fr10a and Fr10b were taken at an interval of 7 months. ${ }^{11}$ Sample Fr11a and Fr11b were taken at an interval of 9 months. ${ }^{12}$ Mutation detected at high frequency in the second sample and identified at low frequency by reviewing the data of the first sample. 
patients by SMRT sequencing are true, physically independent TP53 variants, confirming the results of indirect computational studies currently used to infer cancer heterogeneity. It is highly likely that each TP53 variant belongs to an independent subclone arising from a wildtype TP53 founder clone. The observation of multiple subclones with different TP53 variants in these patients suggests the occurrence of a specific genetic background in the founder clones that requires TP53 inactivation for further progression. All of these subclones present a highly dynamic evolution, but it remains to be determined whether this evolution is driven by treatment, a natural characteristic of the tumor or both. A recent study on 1,514 MDS patients after stem-cell transplantation showed that 283 patients $(19 \%)$ had at least one oncogenic TP53 mutation and a poor overall survival. ${ }^{11}$ One hundred and two $(36 \%)$ of these patients had more than one TP53 variant (range 2-6). It is likely that the use of a sensitive methodology for DNA sequencing will reveal that tumors with multiple TP53 variants constitute a general feature raising potential problems for treatment options. Finally, in this report, we demonstrate the efficiency of SMRT sequencing for the analysis of complex samples. The rapid progress in NGS, combining longer

A

Mar 2008

Lenalidomide $5 \mathrm{mg} /$ day

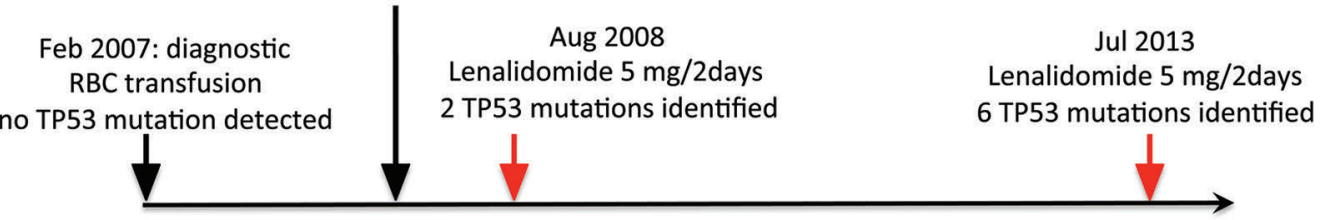

B

Sanger/NGS (Short reads)

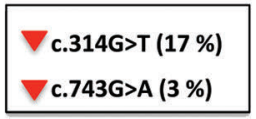

Aug 2008

\section{SMRT sequencing (long reads)}

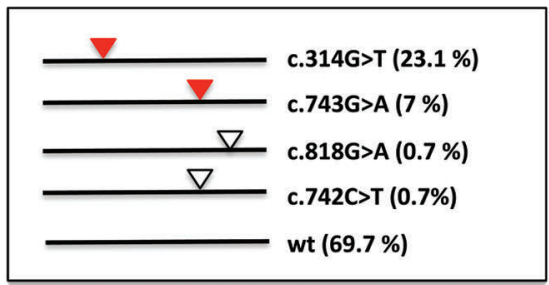

Aug 2008
Jul 2013

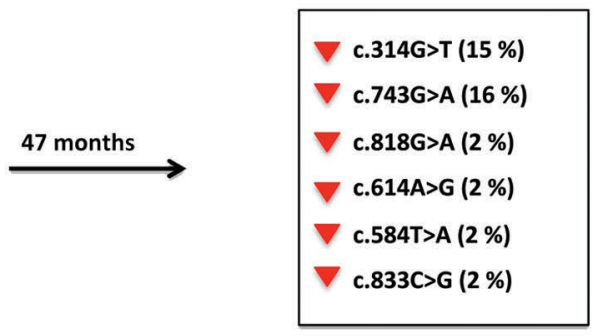


reads, increased sensitivity and decreased costs, will allow investigation of the whole sequence of clinically relevant genes in a single analysis. Long-read RNA-seq analysis could also be used to address the issue of TP53 alternative spliced transcripts that have already been described to be of clinical interest in AML. ${ }^{12}$

Laurence Lodé, ${ }^{1,2}$ Adam Ameur, ${ }^{3}$ Thibault Coste, ${ }^{1,5}$

Audrey Ménard, ${ }^{2}$ Steven Richebourg, 2,4

Jean-Baptiste Gaillard, "Yannick Le Bris, ${ }^{2}$

Marie-Christine Béné, ${ }^{2}$ Thierry Lavabre-Bertrand'

and Thierry Soussi ${ }^{6-8}$

${ }^{1}$ Hematology Laboratory, University Hospital, Montpellier, F-34000, France; ${ }^{2}$ Hematology Laboratory, University Hospital, Nantes, F-44093, France; 'Bepartment of Immunology, Genetics and Pathology, Science for Life Laboratory, Uppsala University, Sweden. ${ }^{4}$ Cytogenetics, Hôpital du Saint Sacrement, CHU de Québec, Université Laval, Department of molecular medicine, Canada; sDepartment of Clinical Cytology and Cytogenetics, Nimes University Hospital, France; ${ }^{6}$ Sorbonne Université, UPMC Univ Paris 06, F75005 Paris, France; ${ }^{7}$ INSERM, U1138, Centre de Recherche des Cordeliers, Paris, France and ${ }^{8}$ Department of Oncology-Pathology, Karolinska Institutet, Cancer Center Karolinska (CCK) R8:04, Stockholm SE-171 76, Sweden

Funding: this work was supported by Radiumhemmets Forskningsfonder and the Swedish Cancer Society (Cancerfonden) to TS. SMRT sequencing was performed by the National Genomics Infrastructure (NGI) hosted by SciLifeLab Uppsala. The authors are most grateful to the Molecular Hematology team and to the IRCNA Tumor Bank (CHU de Nantes, Institut de Cancérologie de l'Ouest, Saint-Herblain F44800, France) for their assistance.

Correspondence: thierry.soussi@ki.se doi:10.3324/haematol.2017.176719

Information on authorship, contributions, and financial \& other disclosures was provided by the authors and is available with the online version of this article at www. haematologica.org.

\section{References}

1. Papaemmanuil E, Gerstung M, Bullinger L, et al. Genomic classification and prognosis in acute myeloid leukemia. N Engl J Med. 2016; 374(23):2209-2221.

2. Jadersten M, Saft L, Smith A, et al. TP53 Mutations in low-risk myelodysplastic syndromes with del $(5 q)$ predict disease Progression. J Clin Oncol. 2011;29(15):1971-1979.

3. Döhner H, Estey E, Grimwade D, et al. Diagnosis and management of AML in adults: 2017 ELN recommendations from an international expert panel. Blood. 2017;129(4):424-447.

4. Malcovati L, Hellström-Lindberg E, Bowen D, et al. Diagnosis and treatment of primary myelodysplastic syndromes in adults: recommendations from the European LeukemiaNet. Blood. 2013; 122(17):2943-2964.

5. Stengel A, Kern W, Haferlach T, Meggendorfer M, Fasan A, Haferlach C. The impact of TP53 mutations and TP53 deletions on survival varies between AML, ALL, MDS and CLL: an analysis of 3307 cases. Leukemia. 2017;31(3):705-711.

6. Mossner M, Jann JC, Nowak D, et al. Prevalence, clonal dynamics and clinical impact of TP53 mutations in patients with myelodysplastic syndrome with isolated deletion $(5 \mathrm{q})$ treated with lenalidomide: results from a prospective multicenter study of the german MDS study group (GMDS). Leukemia. 2016;30(9):1956-1959.

7. da Silva-Coelho P, Kroeze LI, Yoshida K, et al. Clonal evolution in myelodysplastic syndromes. Nat Commun. 2017;815099.

8. Marusyk A, Almendro V, Polyak K. Intra-tumour heterogeneity: a looking glass for cancer? Nat Rev Cancer. 2012;12(5):323-334.

9. Leroy B, Ballinger ML, Baran-Marszak F, et al. Recommended guidelines for validation, quality control, and reporting of TP53 variants in clinical practice. Cancer Res. 2017;77(6):1250-1260.

10. Leroy B, Anderson M, Soussi T. TP53 mutations in human cancer: database reassessment and prospects for the next decade. Hum Mutat. 2014;35(6):672-688.

11. Lindsley RC, Saber W, Mar BG, et al. Prognostic mutations in myelodysplastic syndrome after stem-cell transplantation. $\mathrm{N}$ Engl J Med. 2017;376(6):536-547.

12. Anensen N, Hjelle SM, Van Belle W, et al. Correlation analysis of p53 protein isoforms with NPM1/FLT3 mutations and therapy response in acute myeloid leukemia. Oncogene. 2012;31(12):1533-1545. 